\title{
Radiative Penguin decays at the $B$ Factories
}

\author{
Tülay Çuhadar-Dönszelmann (on behalf of the Babar Collaboration) \\ Department of Physics and Astronomy, University of British Columbia, Vancouver, BC, V6T 1Z1 \\ CANADA
}

\begin{abstract}
Recent results from the $B$-Factories on radiative decays such as $b \rightarrow s(d) \gamma, b \rightarrow s \ell \ell$ and leptonic decay $B^{0} \rightarrow \tau^{+} \tau^{-}$are reviewed.
\end{abstract}

Keywords: Rare decays, Leptonic decays

PACS: $12.15 . \mathrm{Hh}, 13.20 .-\mathrm{v}, 13.20 . \mathrm{He}, 13.30 . \mathrm{Ce}, 13.35 . \mathrm{Dx}$

\section{INTRODUCTION}

The flavor changing neutral current (FCNC) rare $B$ decays such as $b \rightarrow s(d) \gamma$ and $b \rightarrow s \ell \ell$ play a crucial role in testing the Standard Model (SM) as well as probing physics beyond the SM. Such processes proceed via one loop (penguin or box) diagrams. The effective hamiltonian for $b \rightarrow s$ transition is given by $H_{e f f}=-4 \frac{G_{F}}{\sqrt{2}} V_{t s}^{*} V_{t b} \sum_{i} C_{i}(\mu) O_{i}(\mu)[1]$, where $G_{F}$ and $V_{t s, t b}$ are the Fermi constant and the Cabibbo-Kobayashi-Maskawa $(\mathrm{CKM})$ matrix elements, respectively. The FCNC operators, $O_{i}$, can be considered as the vertices of the effective theory and the Wilson coefficients, $C_{i}$, play the role of coupling constants. Contributions in the loops of these decays from the Higgs boson or supersymmetric particles can lead to an enhancement in their rates which range between $10^{-4}$ to $10^{-6}$ in the SM. A comparison between the measurement and the SM prediction provides a stringent constraint on such new particles. At lowest order $b \rightarrow s(d) \gamma$ is described by the coefficient $C_{7}$ (photon penguin operator) and is computed up to next-toleading order (NLO). The decay $b \rightarrow s \ell \ell$ is defined by a $Z$-penguin and a $W$-box diagram in addition to the radiative penguin. Its amplitude depends on the coefficients $C_{7}, C_{9}$ and $C_{10}$ (vector and axial vector operators) which are computed next-to-next-to-leading order (NNLO).

The Babar and Belle detectors are located at the PEP-II and KEKB asymmetric energy $e^{+} e^{-}$colliders, respectively. $B \bar{B}$ pairs are produced coherently from the $\Upsilon(4 S)$ decays. Up to now, approximately $400 \mathrm{fb}^{-1}$ and $600 \mathrm{fb}^{-1}$ of data have been recorded by the Babar and Belle experiments, respectively.

$$
b \rightarrow s \gamma
$$

The signature of a $b \rightarrow s \gamma$ decay is an energetic photon of $E_{\gamma}^{*}=(2-2.7) \mathrm{GeV}$. Due to the confinement of the $b$-quark inside the $B$ meson, the energy spectrum is smeared instead of producing a monochromatic energy of $E \approx m_{b} / 2$. A fit to $E_{\gamma}^{*}$, or to the first and second moments of the photon energy spectrum, can be used to extract information on the Heavy Quark Expansion (HQE) parameters, $m_{b}$ ( $b$-quark mass) and $\mu_{\pi}^{2}$ ( $b$-quark 
kinetic energy inside the $B$ meson). The $E_{\gamma}$ spectrum is also input to semileptonic $B$ decay analyses to extract $\left|V_{c b}\right|$ and $\left|V_{u b}\right|$. The inclusive decay $B \rightarrow X_{s} \gamma$ is studied with two methods. In the fully inclusive method, the photon energy spectrum is measured without reconstructing the $X_{s}$ system. In the semi exclusive method, the $X_{s}$ system is reconstructed from a kaon ( $K$ or $K_{S}$ ) and up to four pions (one or two $\pi^{0}$ allowed). The reconstructed $X_{S}$ system is then combined with a photon to form a $B$ meson.

Babar and Belle have measured the branching fraction and the moments from the photon energy spectra using $82 \mathrm{fb}^{-1}$ and $140 \mathrm{fb}^{-1}$, respectively [2, 3]. The branching fraction results are summarized in Fig.1(left) which also includes the earlier measurement by CLEO at $9.1 \mathrm{fb}^{-1}$ [4]. The world average of $B(b \rightarrow s \gamma)=(3.55 \pm 0.26) \times 10^{-4}$ is published by the Heavy Flavor Averaging Group (HFAG) [5]. It is in good agreement with the SM prediction of $B(b \rightarrow s \gamma)=\left(3.61_{-0.49}^{+0.37}\right) \times 10^{-4}$ [6]. A fit to all available $b \rightarrow s \gamma$ and $B \rightarrow X_{c} \ell v$ data results in the HQE parameters of $m_{b}=4.590 \pm 0.039 \mathrm{GeV}$ and $\mu_{\pi}^{2}=0.401 \pm 0.040 \mathrm{GeV}^{2}$ (in the kinetic scheme) [7]. As shown in Fig.1(right), the moments obtained from the $E_{\gamma}$ spectra are consistent among the different measurements as well as with the theory predictions that rely on data from $B \rightarrow X_{c} \ell v$ moments. The $b \rightarrow s \gamma$ decay mode has also been measured in many exclusive final
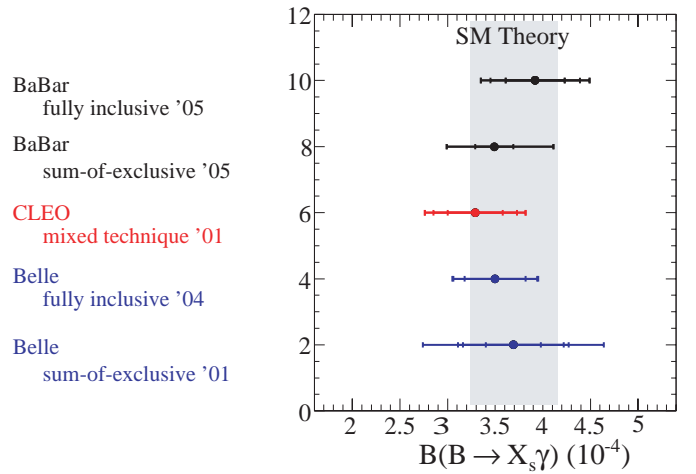
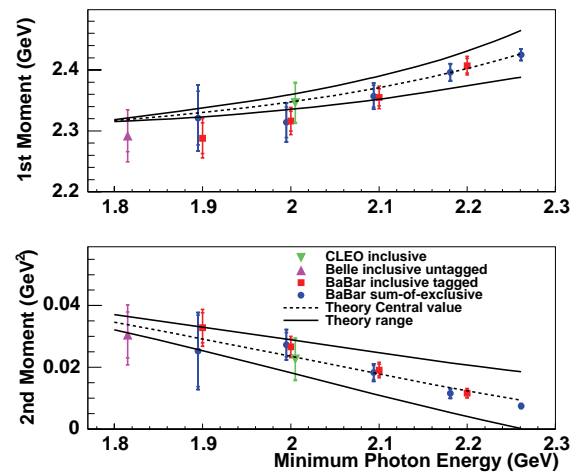

FIGURE 1. (left) Results on branching fraction of $b \rightarrow s \gamma$. (right) First and second moments from photon spectra of $b \rightarrow s \gamma$.

states. For example, the most recent Babar result on $B \rightarrow K \eta \gamma$ confirms the earlier Belle measurement. Both charged and neutral decays are observed at better than $5 \sigma$ significance. The combined average limits are $B\left(B^{+} \rightarrow K^{+} \eta \gamma\right)=(9.3 \pm 1.1) \times 10^{-6}$, and $B\left(B^{0} \rightarrow K^{0} \eta \gamma\right)=\left(10.3_{-2.1}^{+2.3}\right) \times 10^{-6} \quad$ [5]. Babar has also reported an upper limit $B\left(B^{+(0)} \rightarrow K^{+(0)} \eta^{\prime} \gamma\right)<4.2(6.6) \times 10^{-6}$ at the $90 \%$ C.L. [8].

$$
b \rightarrow d \gamma
$$

The decay $b \rightarrow d \gamma$ has similar sensitivity to the new physics (NP) as $b \rightarrow s \gamma$, but its branching fraction is suppressed by the ratio of $\left|V_{t d} / V_{t s}\right|^{2}$. The exclusive decay $B \rightarrow$ $(\rho, \omega) \gamma$ were first observed by Belle using $386 \times 10^{6} B \bar{B}$. Assuming isospin symmetry, a simultaneous fit to three decay modes $\left(B \rightarrow\left(\rho^{+}, \rho^{0}, \omega\right) \gamma\right)$ results in $B(B \rightarrow(\rho, \omega) \gamma)=$ $\left(1.32_{-0.31}^{+0.34}(\text { stat })_{-0.09}^{+0.10}(\right.$ sys $\left.)\right) \times 10^{-6}$ [9]. Based on $211 \times 10^{6} B \bar{B}$ events, Babar observed no evidence for these processes and placed combined upper limits of $B(B \rightarrow(\rho, \omega) \gamma)<$ 
$1.2 \times 10^{-6}$ at the $90 \%$ C.L. [10]. The SM predictions for these branching fractions are in the range of $(0.8-1.8) \times 10^{-6}$ [11]. The UTfit collaboration has performed a fit to the $b \rightarrow d \gamma$ data to extract the $\left|V_{t d} / V_{t s}\right|$ ratio. The fit results in the value of $\left|V_{t d} / V_{t s}\right|=0.16 \pm 0.02[12]$. The most recent value of $\left|V_{t d} / V_{t s}\right|=0.208_{-0.007}^{+0.008}$ is reported by CDF Collaboration [13] from the $\Delta m_{s} / \Delta m_{d}$ measurement.

$$
b \rightarrow s \ell \ell
$$

In addition to the decay rate of $b \rightarrow s \ell \ell$, other observables such as the forward-backward asymmetry $\left(A_{F B}\right)$ can be used to identify the sign of the Wilson coefficient $C_{7}$ and the magnitudes of $C_{9}$ and $C_{10}$. The CP asymmetry, $A_{C P}$, and the ratio of the muon to electron rate, $R_{K^{(*)}}=B\left(B \rightarrow K^{(*)} \mu \mu\right) / B\left(B \rightarrow K^{(*)} e e\right)$, are sensitive to NP.
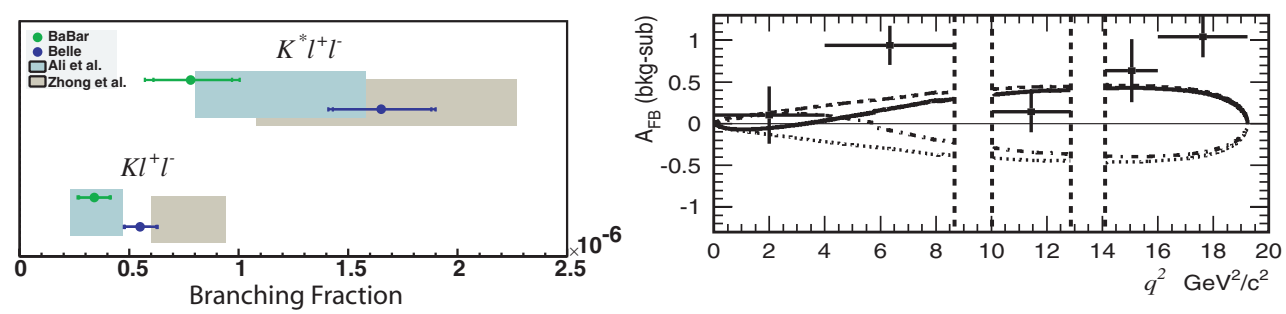

FIGURE 2. (left) Comparison of the $B \rightarrow K^{(*)} \ell \ell$ branching fraction measurements and the two SM predictions. (right) $A_{F B}$ in $B \rightarrow K^{*} \ell \ell$ measured by Belle Collaboration. The solid line is for negative $A_{7}$, dashed line is for positive $A_{7}$, dot-dashed is for positive $A_{10}$ and dotted is for positive $A_{7}$ and $A_{10}$.

Semi exclusive decay $b \rightarrow s \ell \ell$ is reconstructed from two leptons $(\ell=e, \mu)$, a kaon and up to four pions (one or two $\pi^{0}$ allowed). Babar and Belle have measured the branching fraction summing up 10 and 18 hadronic states $[14,15]$, respectively. In order to avoid the resonance regions, the branching fraction has been reported in two $q^{2}$ (dilepton mass squared) regions: $q^{2}=[1-6] \mathrm{GeV}^{2}$ and $q^{2}>14 \mathrm{GeV}^{2}$. For $q^{2}=[1-6]$ $\mathrm{GeV}^{2}$, branching fraction measured by Babar [Belle] $B(B \rightarrow s \ell \ell)=(1.8 \pm 0.9)[1.5 \pm$ $0.6] \times 10^{-6}$ is consistent with the SM prediction of $(1.6 \pm 0.2) \times 10^{-6}$ [16]. For $q^{2}>16$ $\mathrm{GeV}^{2}$, the branching fraction results from Babar and Belle are $(5.6 \pm 2.0) \times 10^{-6}$ and $(4.1 \pm 1.1) \times 10^{-6}$, respectively. These result agree well with the SM expectation of $(4.4 \pm 0.7) \times 10^{-6}[16]$.

The exclusive decay $B \rightarrow K^{(*)} \ell \ell$ has been studied extensively by Babar [17] and Belle [18] using $208 \mathrm{fb}^{-1}$ and $253 \mathrm{fb}^{-1}$ of data. The measured branching fractions are summarized on Fig.2(left). The CP asymmetries, $A_{C P}^{K}=(-0.07 \pm 0.22)$ and $A_{C P}^{K^{*}}=$ $(0.03 \pm 0.23)$ measured by Babar are very small, consistent with the SM expectation. For the ratio, Babar has reported $R_{K}=(1.06 \pm 0.49)$ and $R_{K^{*}}=(0.91 \pm 0.45)$. For these ratios, Belle obtains $R_{K}=(1.38 \pm 0.41)$ and $R_{K^{*}}=(0.98 \pm 0.32)$. The results of $R_{K}$ and $R_{K^{*}}$ agree well with the SM expectation of unity [19] and 0.75 [20], respectively.

The $A_{F B}\left(q^{2}\right)$ measurement from Belle [21] is shown in Fig.2(right). The plot shows NP scenarios as well as the SM one. The Belle results exclude the NP scenarios where the sign of $C_{9} C_{10}$ is flipped with respect to the SM. The models with $C_{7}$ and $-C_{7}$ agree well with data, hence no conclusion can be made on the sign of $C_{7}$. Babar has measured $A_{F B}$ in two regions. At large $q^{2}$, the $C_{9} C_{10}$ is found to be consistent with the SM. At 
low $q^{2}$, a lower limit $A_{F B}>0.19$ at $95 \%$ C.L. has been set. The fraction of longitudinal polarization, $F_{L}$, in the $K^{*}$ mode has the same importance as $A_{F B}$ in measuring the sign of $C_{7}$. Babar has measured $F_{L}$, however data does not favor either of the $C_{7}$ sign.

\section{LEPTONIC DECAYS $B^{0} \rightarrow \tau^{+} \tau^{-}$}

The recent result from Babar on $B^{0} \rightarrow \tau^{+} \tau^{-}$is also presented. This decay proceeds via $W$-box and penguin diagrams. Due to the large $\tau$ mass, the branching fraction is less suppressed with respect to electron and muon modes $\left(e: \mu: \tau=10^{-7}: 10^{-3}: 1\right)$. However, the multiple neutrinos in the final states make it harder to measure. The SM prediction is $B\left(B^{0} \rightarrow \tau^{+} \tau^{-}\right)=1.2 \times 10^{-7}\left[f_{B} / 200 \mathrm{MeV}\right]^{2}\left[\left|V_{t d}\right| / 0.007\right]^{2}$ where $f_{B}$ is the $B$ meson decay constant [1]. Using $210 \mathrm{fb}^{-1}$, no evidence was found for this decay. At the $90 \%$ C.L., the upper limit of $B^{0} \rightarrow \tau^{+} \tau^{-}<4.1 \times 10^{-3}$ has been obtained [22].

\section{CONCLUSION}

Such rare decays, pioneered by CLEO, are brought to a more mature state by the $B$-Factories. The results shown here are based on one-fourth to one-half of the total integrated luminosity. Both theoretical and experimental updates will further constraint physics beyond the SM.

\section{REFERENCES}

1. P. Harrison and H. Quinn, Tech. Rep. SLAC-R-504, SLAC (1998).

2. BABAR Collaboration, B. Aubert et al., hep-ex/0507071; Phys. Rev. D 72, 052004 (2005).

3. Belle Collaboration, P. Koppenburg et al., Phys. Rev. Lett. 93, 061803 (2004); K. Abe et al., Phys. Lett. B 511, 151 (2001).

4. CLEO Collaboration, S. Chen et al., Phys. Rev. Lett. 87, 251807 (2001).

5. E. Barberio et al., hep-ex/0603003; online update at http://www.slac.stanford.edu/xorg/hfag .

6. T. Hurth et al., Nucl. Phys. B 704, 56 (2005).

7. O. Buchmüller and H. U. Flächer, Phys. Rev. D 73, 073008 (2006).

8. BABAR Collaboration, B. Aubert et al., hep-ex/0603054, submitted to Phys. Rev. Lett.

9. Belle Collaboration, D. Mohapatra et al., Phys. Rev. Lett. 96, 221601 (2006).

10. BABAR Collaboration, B. Aubert et al., Phys. Rev. Lett. 94, 011801 (2005).

11. A. Ali et al., Phys. Lett. B 595, 323 (2004); S. W. Bosch and G. Buchalla Nucl. Phys. B 621, 459 (2002).

12. UTfit Collaboration, M. Bona et al., http://utfit.roma1.infn.it/btovg/ckm-btovg.html.

13. Talk by Guillelmo Gomez-Ceballos, "Update on CDF $B_{s}$ Mixing", Flavor Physics and CP Violation conference, CANADA, 2006.

14. BABAR Collaboration, B. Aubert et al., Phys. Rev. Lett. 93, 081802 (2004).

15. Belle Collaboration, M. Iwasaki et al., Phys. Rev. D 72, 092005 (2005).

16. P. Gambino, U. Haisch and M. Misiak, Phys. Rev. Lett. 94, 061803 (2005).

17. BABAR Collaboration, B. Aubert et al., Phys. Rev. D 73, 092001 (2006).

18. Belle Collaboration, K. Abe et al., hep-ex/0410006 .

19. G. Hiller and F. Krüger Phys. Rev. D 69, 074020 (2004).

20. A. Ali et al.Phys. Rev. D 66, 034002 (2002).

21. Belle Collaboration, A. Ishikawa et al., Phys. Rev. Lett. 96, 251801 (2006).

22. BABAR Collaboration, B. Aubert et al., Phys. Rev. Lett. 96, 241802 (2006). 